\title{
The Optimal Stimulation Pattern for Skeletal Muscle Is Dependent on Muscle Length
}

\author{
Petra Mela, Peter H. Veltink, Member, IEEE, Peter A. Huijing, Stanley Salmons, and Jonathan C. Jarvis
}

\begin{abstract}
Stimulation patterns can be optimized by maximizing the force-time integral (FTI) per stimulation pulse of the elicited muscle contraction. Such patterns, providing the desired force output with the minimum number of pulses, may reduce muscle fatigue, which has been shown to correlate to the number of pulses delivered. Applications of electrical stimulation to use muscle as a controllable biological actuator may, therefore, be improved. Although muscle operates over a range of lengths, optimized patterns have been determined only at optimal muscle length. In this study, the patterns with up to four pulses that produced the highest isometric FTI were determined at 10 muscle lengths for 11 rabbit tibialis anterior muscles. The interpulse intervals (IPIs) used ranged from 4 to $54 \mathrm{~ms}$. At high muscle length, the optimal stimulation pattern consisted of an initial short IPI (doublet) followed by longer IPIs, in agreement with previous studies. However, at low length, the third pulse still elicited more than linear summation (triplet); furthermore, the relative enhancement of the FTI per pulse was considerably larger at low length than at high length, suggesting that optimal stimulation patterns are length dependent.
\end{abstract}

Index Terms-Electrical stimulation, muscle length, $n$-lets stimulation pattern.

\section{INTRODUCTION}

$\mathbf{S}$ KELETAL muscle is a highly complex, nonlinear system capable of converting chemical energy into mechanical work. The process can be artificially triggered with only a small expenditure of energy needed to activate the motor nerve.

Skeletal muscle has, therefore, attracted interest as controllable biological force generator in a number of clinical applications in which its activation is provided artificially by means of electrical stimulation (e.g., [33], [13], [32], [27], [29]).

When a muscle is activated by means of electrical stimulation, however, the size principle that determines the order of recruitment of motor units under normal physiological conditions does not apply. Normally, the smaller, oxidative motor units have

Manuscript received February 12, 2001; revised March 27, 2002. This work was supported by the Training and Mobility of Researchers Program of the European Union (NEUROS Project).

P. Mela and P. H. Veltink are with the Institute for Biomedical Technology (BMTI), Biomedical Signals and Systems, Department of Electrical Engineering, University of Twente, 7500 AE Enschede, The Netherlands (e-mail: p.mela@el.utwente.nl; p.h.veltinnk@el.utwente.nl).

P. A. Huijing is with the Institute for Biomedical Technology (BMTI), Biomedical Signals and Systems, Department of Electrical Engineering, University of Twente, 7500 AE Enschede, The Netherlands, and also with the Faculteit Bewegingswetenschappen, Vrije Universiteit Amsterdam, 1081 BT Amsterdam, The Netherlands (e-mail: p_a_j_b_m_huijing@fbw.vu.nl).

S. Salmons and J. C. Jarvis are with the British Heart Foundation Skeletal Muscle Assist Research Group, Department of Human Anatomy and Cell Biology, University of Liverpool, Liverpool L69 3GE, U.K. (e-mail: s.salmons@ liverpool.ac.uk; jcj@liverpool.ac.uk).

Publisher Item Identifier S 1534-4320(02)05946-6. the lowest threshold for activation. The fast-fatiguable fibers are generally recruited at higher force levels and therefore less often. When muscles are stimulated by applying an electrical field across their motor nerve, the situation is reversed and the large motor neurons supplying the fast-fatiguable motor units have the lowest threshold for activation [16], [26]. Furthermore, axons are activated synchronously, and therefore the benefits of asynchronous activation [25] within the motor unit pool are not present. With functional electrical stimulation (FES), then, higher rates of motor unit activation are needed to produce fused tetani, and the rate of fatigue is higher than normal at submaximal force [10], [2], [30]. Furthermore, following spinal cord injury, paralyzed muscle is much more fatiguable because inactive fibers tend to transform to the fast phenotype [8].

Fatigue is thus a major problem in FES of muscle. Muscle training can certainly help, but also has the potential for unwanted slowing [17]. Since both fatigue in the short term and slowing in the longer term is correlated to the number of pulses delivered to the neuromuscular system [21], [14], [28], [30], optimal patterns of stimulation have been investigated in a variety of applications with the aim of eliciting a desired force output with the minimum number of pulses [4], [12], [18], [20], [22], [3]. A possible approach to determine optimum patterns of stimulation is to maximize the force-time integral (FTI) per unit impulse (FTIpP) of the elicited isometric contractions. An optimal stimulation sequence can be determined with an iterative method by identifying the interpulse interval (IPI) between the first two pulses (doublet) which produces the maximum FTIpP. That IPI is then held constant, a third pulse is added to the sequence, and the new IPI optimized in the same way. The procedure is repeated for an increasing number of pulses, every time with only the final IPI being varied and all prior IPIs being held constant at their previously determined optimal values [9], [34], [20], [18].

It has been shown that the resulting optimized sequences for fast muscle consist of two initial pulses separated by a very short IPI (doublet), followed by pulses at longer IPIs [9], [34], [24], [20]. The more-than-linear enhancement of force output due to the second of the two initial pulses is referred to as the "doublet effect."

Although a comprehensive explanation of the doublet effect is not yet available, it is likely that the length of the muscle for which the stimulation train is delivered will affect the results. Despite this likelihood and the fact that muscle works over a range of lengths, optimal stimulation patterns have been determined usually at one muscle length exclusively, commonly the optimum length, i.e., the length at which the muscle develops the highest isometric twitch force [9], [34], [24], [20]. 
The purpose of this study was to investigate the length dependence of optimal four pulse stimulation patterns.

\section{MATERIALS AND METHODS}

\section{A. Setup and Animal Preparation}

The experiments were carried out on the tibialis anterior muscle of rabbits under fentanyl/fluanisone anaesthesia with mechanical ventilation $(n=6)$. Cuff electrodes (bipolar electrodes with an insulating cover) were placed around the common peroneal nerves of both legs, and the nerves were transected proximal to the electrode site to isolate the nerve-muscle preparation from the central nervous system. Data were collected from 11 muscles because of surgical damage to the peroneal nerve of one leg during one of the animal preparations. The fine attachments between the tibialis anterior muscle and the extensor digitorum longus muscle were divided, taking care not to disturb the neurovascular pedicle on the underside of the tibialis anterior muscle. The tibialis anterior tendon was transected in the foot and held in a miniature titanium alloy clamp, leaving a minimum of unclamped tendon. The alloy clamp was connected by a lightweight, noncompliant carbon fiber-epoxy link to the arm of a Dual Mode Servo System, a force and length controller designed specifically for muscle measurements (Cambridge Technology, Inc., Watertown, MA). A personal computer was used to provide synchronized control of the servo motor, muscle stimulation, and data acquisition (sampling frequency $1000 \mathrm{~Hz}$ ), via a commercial input/output (I/O) board (National instruments Corporation, Austin, TX). The code was written in Labview (National instruments Corporation, Austin, TX) and Matlab (The MathWorks, Inc.). The stimulus pulses were generated by an isolated stimulator (Mk. IV, Devices, Hertfordshire, U.K.), which was triggered by the PC via a first-in/first-out (FIFO) buffer to ensure that the operation of the computer did not degrade the precision of the programmed IPIs. The pulses delivered to the muscle were $200 \mu \mathrm{s}$ long, and the voltage was set at three times the threshold for force generation, which provided reliable activation of all the motor units in the tibialis anterior muscle.

\section{B. Iterative Method}

The muscle length range for the experiments extended from the lowest length at which active twitch force could be registered to the length at which passive force rose to a value of approximately $4 \mathrm{~N}$ (Fig. 1), which was considered a safe limit to avoid muscle tissue damage. The optimum muscle length was determined by delivering twitches at 10 lengths evenly distributed within the length range. To be able to compare results from muscles belonging to different animals despite interanimal differences, the 10 lengths were considered as length categories from 1 to 10,1 corresponding to the lowest and 10 the highest muscle length. We have used the term muscle length category when referring to mean results from all the muscles. A clear optimum in the twitch force-length curve was usually not reached and the highest tested length was considered optimum length.

In each experiment, at the chosen lengths, doublets with various IPIs $(2,4,6,8,12,24$, and $42 \mathrm{~ms})$ were delivered for a

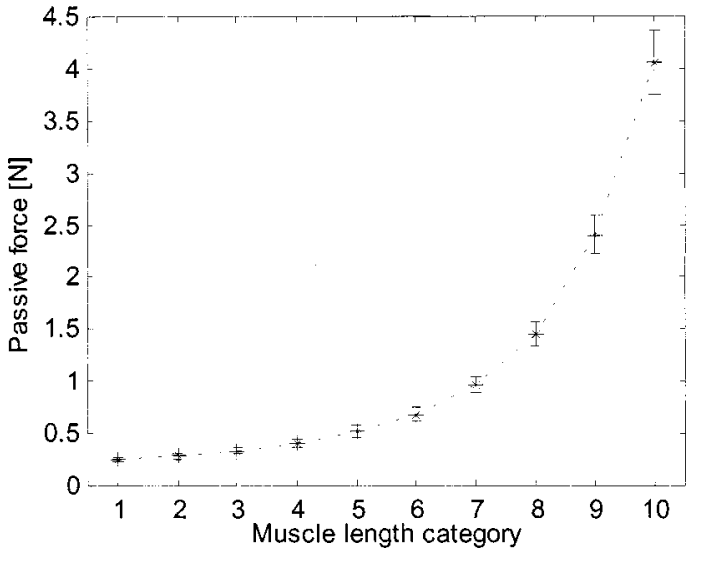

Fig. 1. Passive force at 10 length. Presented are mean values $(n=6) \pm$ standard error of mean.

total of 70 contractions. One minute was allowed between contractions for recovery. The sequence was randomized for muscle length and IPI in order to avoid systematic fatigue effects on the results. Fatigue effects on a specific IPI sequence at a specific length would then be canceled out when averaging over all the tested muscles. For each doublet, isometric force was measured, FTI calculated and plotted against the interval between the two pulses. For each muscle length, the IPI which gave the highest FTI was selected and in a second randomized sequence an additional pulse was added to the train at IPIs of 2, 4, 8, 16, 24, 28, $32,36,42,54 \mathrm{~ms}$ to find the optimal triplet pattern. The optimal IPI of the third pulse was, then, determined with the same criterion and a third randomized sequence performed to determined optimal quadruplets with IPIs of $8,12,24,32,42,54 \mathrm{~ms}$. The number of IPIs to be tested in this last sequence was limited to six in order to limit the duration of the experiment and avoid the occurrence of excessive fatigue.

When different IPIs produced equal FTI, the longest IPI was chosen as to have a longer time and force production with the same number of pulses.

\section{Data Processing}

If the muscle behaved as a linear system, the FTI of its response would increase in direct proportion to the number of pulses applied and the ratio between the FTI of the response to an $n$-pulse train and $n$ times the FTI of a single twitch (normalized FTIpP : nFTIpP) would be unity.

An nFTIpP greater than unity represents a more than linear summation and it is an index of the effectiveness of the stimulation train.

1) Fatigue Index: The experiment consisted of 10 twitch contractions, followed by 70 doublets, 100 triplets, and 60 quadruplets.

Although 1 min was allowed for recovery after each contraction and the sequence was randomized, the duration of the experiment required a regular assessment of the level of fatigue of the muscle. In fact, to calculate the nFTIpP of each contraction the FTI of a twitch at the corresponding muscle length was used. Twitch FTIs at the 10 lengths were, however, determined at the beginning of the experiment and not repeated. An estimate of 


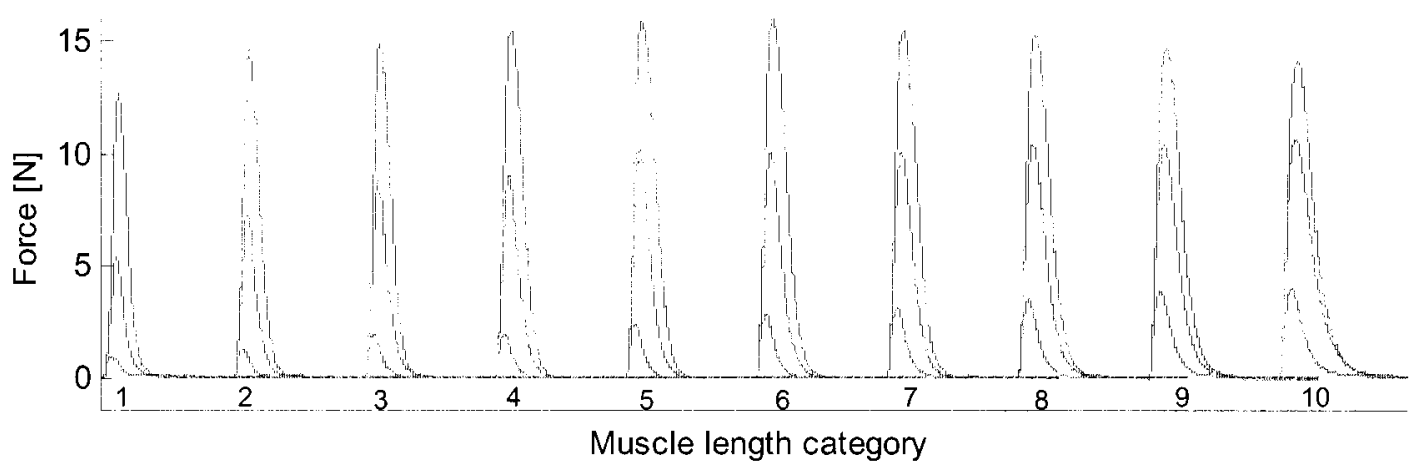

Fig. 2. Muscle responses to twitches, doublets, and triplets at different muscle lengths after subtracting passive force. The pulses of the doublets and the triplets were separated by $4 \mathrm{~ms}$.

twitch FTI at each length throughout the experiment was therefore needed.

Every $10 \mathrm{~min}$, a protocol consisting of a twitch followed by a $0.3-\mathrm{s} 40-\mathrm{Hz}$ tetanus and a second twitch was delivered at optimum length. A fatigue index was calculated by determining the FTI of the pretetanic twitch of the fatigue tests and dividing it by the FTI of the twitch elicited at the beginning of the experiment. Each FTI of doublets and triplets was, respectively, divided by two or three times the twitch FTI at the corresponding length multiplied by the fatigue index estimated from the closest fatigue test.

2) Statistical Analysis: Since the interanimal variation is reported to be greater than the side-to-side variation in individual animals [1], the results (nFTIpP) of the left and right muscle of the same animal were considered as repeated measurements and therefore averaged. Mean values and standard errors have been determined on six sets of data, one for each rabbit.

Two-way ANOVA for repeated measures was used to test for significant differences of $\mathrm{nFTIpP}(p<0.05)$ for IPI and muscle length category.

\section{RESULTS}

Fig. 2 shows typical superimposed experimental traces of muscle force in response to single twitches, doublets, and triplets (all IPIs $=4 \mathrm{~ms}$ ) at 10 muscle lengths. For such a short IPI, the response to doublets and triplets, at all lengths, fused into single twitch-like force-time traces, of which the amplitudes greatly exceeded those of a single pulse and were much greater than expected for linear summation of twitch responses.

\section{A. Doublets}

Fig. 3 illustrates muscle force in response to two pulses delivered at different IPIs for two muscle lengths toward the extremes of the length range and one muscle length approximately in the middle of that range. The traces belong to the same experiment shown in Fig. 2, corresponding to muscle length category 3,6 , and 10. In all cases, the response to the doublet with IPI $=2 \mathrm{~ms}$ was similar to a single twitch, probably because such IPI fell within the refractory period and did not elicit any muscle reaction. Longer IPIs ( 24 and $42 \mathrm{~ms}$ ) produced two fused but distinguishable twitches, the second of which had the higher amplitude. Note the dependence on muscle length of this effect and how $42 \mathrm{~ms}$ between pulses resulted in two almost separate twitches at low muscle length, while the summation was still considerable at high muscle length.

The force-time traces as shown in Fig. 3 have not been corrected for any fatigue effects: this, together with possible experimental variations, may explain why the first parts of the contractions are not perfectly coincident.

The IPI that produced the highest peak force as well as FTI was $4 \mathrm{~ms}$ at all lengths. However, the effect of the second pulse decreased with increasing muscle length. It produced approximately a four-fold increase in peak force above a single twitch at low length [Fig. 3(a)] and a 2.5-fold increase at high length [Fig. 3(c)].

Mean results of the nFTIpP for doublets at low, intermediate, and high muscle length are presented in Fig. 4(a).

The pattern of summation with respect to IPI was similar at the three muscle lengths, resulting in a close to exponential trend of the experimental nFTIpP plotted against IPI. At low muscle length, on average a doublet with short IPI (4 ms) produced FTI three times that produced by two separate twitches at the same muscle length. At high muscle length, the nFTIpP was lower, but still considerably higher than the unity for low IPIs, apart from the 2-ms IPI for which the nFTIpP was 0.5 as it did not produce any summation.

Fig. 4(b) shows muscle length effect on the nFTIpP elicited with three different IPIs (4, 24, and $42 \mathrm{~ms})$.

For all muscle lengths, the optimum IPI is approximately $4 \mathrm{~ms}$ and the pattern of length-dependent summation is similar. The magnitude of potentiation due to the second pulse decreased continuously with increasing muscle length for all IPIs. A two-way repeated measures analysis of variance shows significant influence of muscle length category and IPI on nFTIpP and significant interaction of the two factors.

\section{B. Triplets}

At each length, a third pulse was added to the optimal doublet.

As for the doublets at low muscle lengths, a short IPI produced a contraction with much higher peak force and FTI, the effect being clearly dependent on the interval between the second 


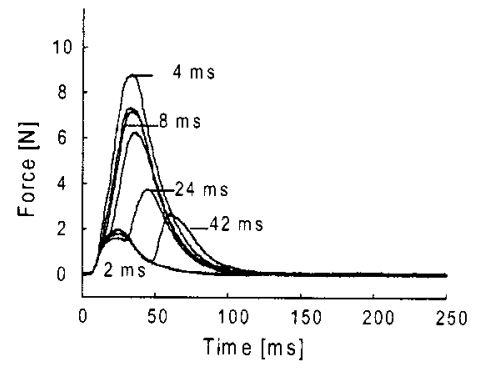

a)

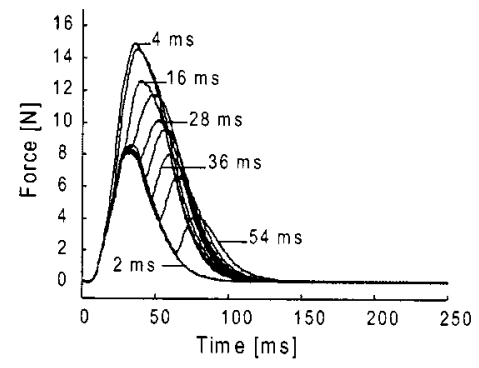

d)

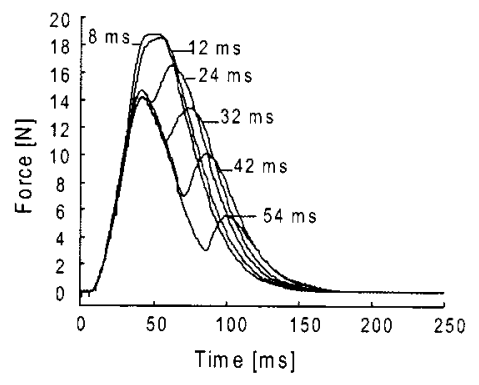

g)

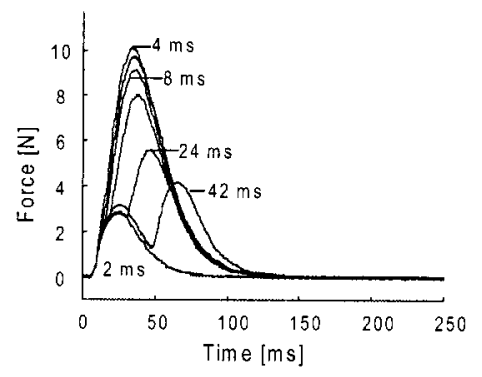

b)

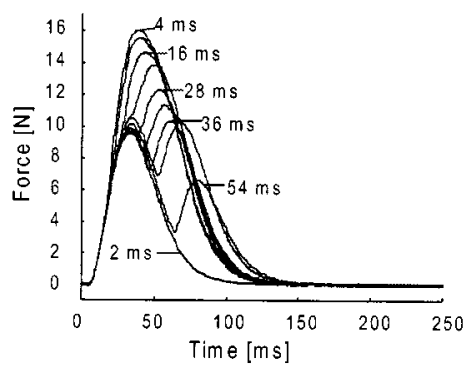

e)

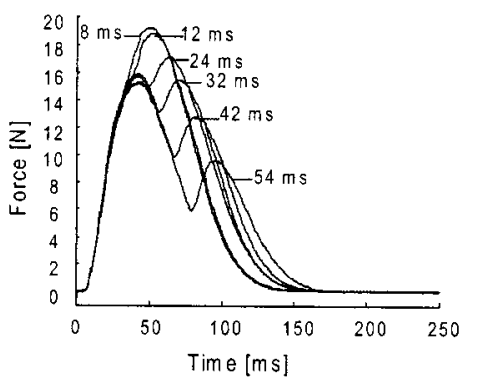

h)

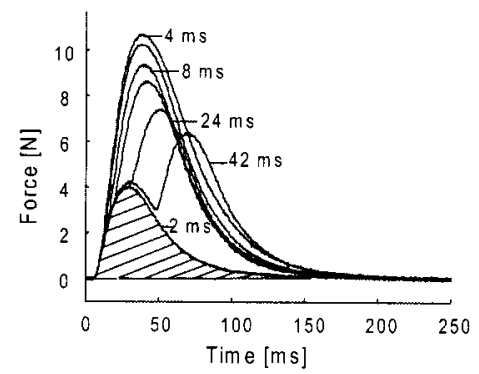

c)

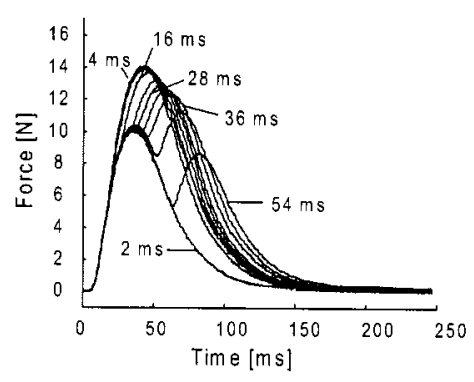

f)

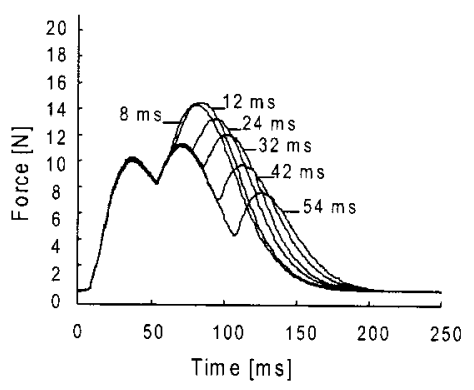

i)

Fig. 3. Muscle responses to two, three, and four pulse trains at (a), (d), (g) low, (b), (e), (h) intermediate, and (c), (f), (i) high muscle length. (c) The hatched area represents the FTI of the doublet elicited with 2-ms IPI. Note that each $n$-pulse train was built on the optimized $n-1$ pulse train. The IPIs indicated in each figure are the intervals between the last two pulses of the train. For two [(a)-(c)] and three [(d)-(f)] pulse trains only some of the used IPIs are indicated for clarity reasons. Shorter IPIs result in higher peak forces and shorter contraction durations.

and the third pulse [Fig. 3(d)]. The contribution of the third pulse to FTI is clearly diminishing at increasing muscle length and at high muscle length [Fig. 3(f)], the addition of a third pulse was not as beneficial and the difference in FTIs corresponding to different IPIs not as appreciable; note that force-time traces at IPI $=2 \mathrm{~ms}$ correspond to the optimal doublets since no summation occurred.

Summation depends more on IPI for low length than for high length. At low muscle length [Fig. 4(c)], the pattern of length dependence of summation resembles that obtained for doublets, while, at high muscle length, the pattern is clearly different: the $n F T I p P$ changed little with increasing IPIs and a maximum was achieved for a 42-ms IPI.

A two-way repeated measures analysis of variance shows significant influence of muscle length category and IPI on nFTIpP and significant interaction of the two factors.

\section{Quadruplets}

After optimal triplets were determined, a fourth pulse was added to the pulse train and the same iterative procedure repeated. Six IPIs $(8,12,24,32,42,54 \mathrm{~ms})$ were used at 10 muscle lengths [Fig. 3(g)-(i)]. It can be noticed that the results of the quadruplets [Fig. 4(e) and (f)] was more variable since it was measured at the end of the experiment where more fatigue occurred. However, fairly reproducible results in term of nFTIpP were obtained. It is clear from Fig. 4(e) that different IPIs produced very similar FTIpP at the three length categories. Accordingly, a two-way repeated measures ANOVA on results for all muscle lengths and IPIs shows significant influence only of muscle length category on nFTIpP.

Summarizing, Fig. 5 shows a three-dimensional representation of mean nFTIpP of doublets, triplets, and quadruplets as a 


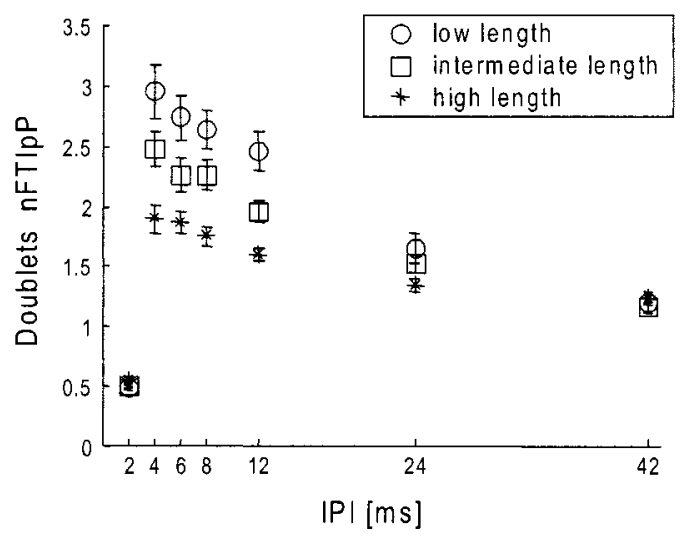

a)

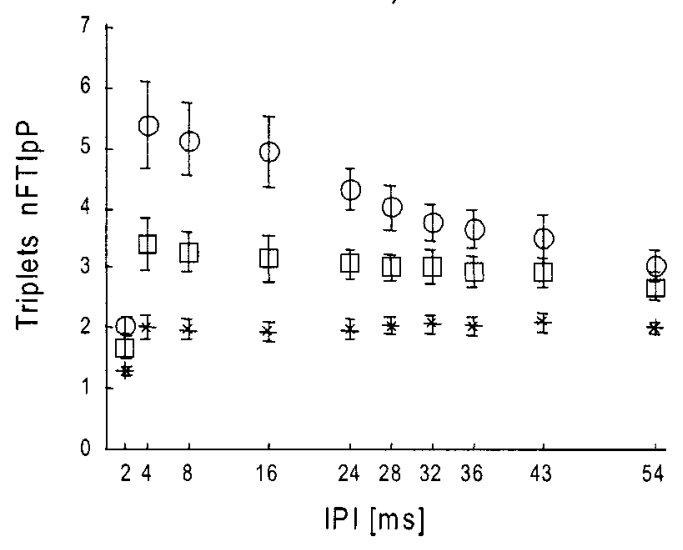

c)

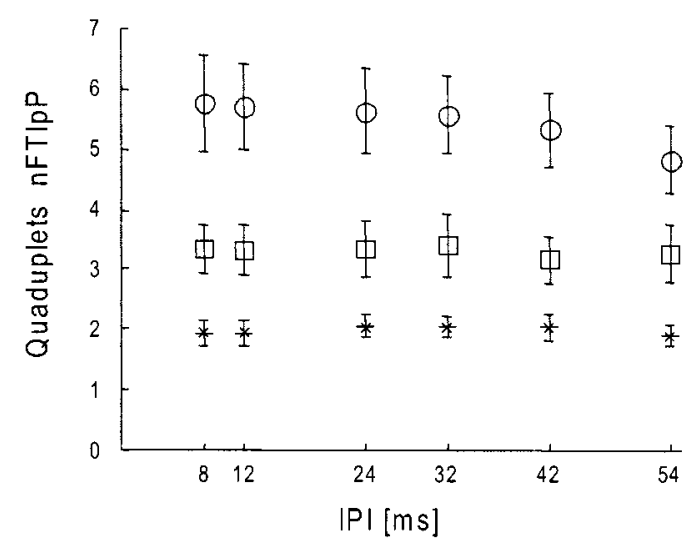

e)

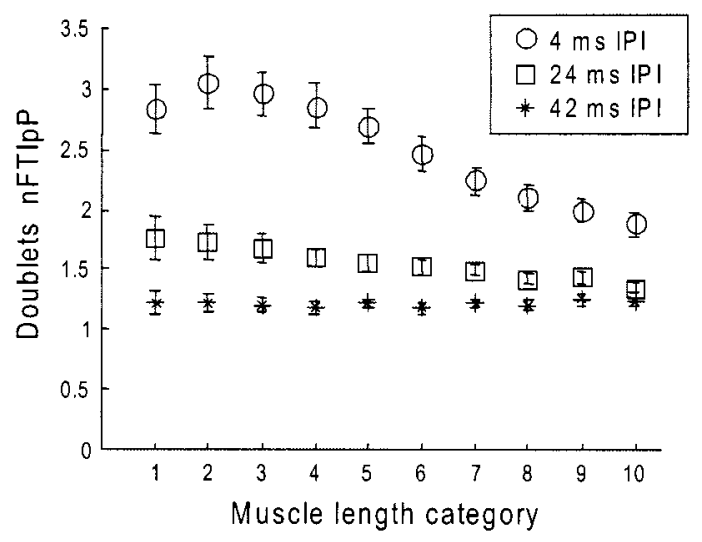

b)

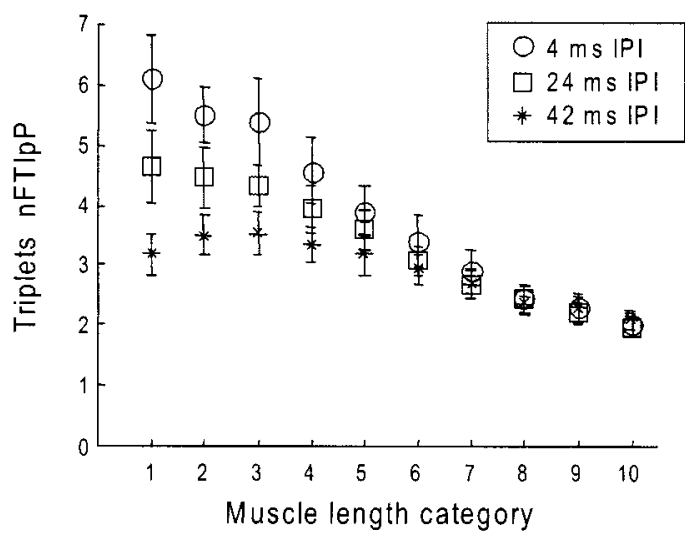

d)

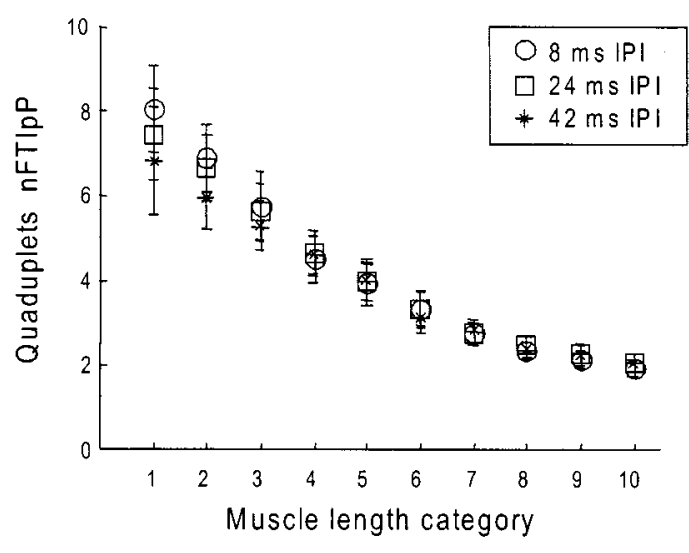

f)

Fig. 4. Mean nFTIpP $(n=6)$ for (a) doublets, (c) triplets, (e) quadruplets at low (circle), intermediate (square), and high (star) muscle length as a function of IPI. Low, intermediate, and high lengths correspond, respectively, to length categories 3, 6, and 10. Mean nFTIpP ( $n=6$ ) for (b) doublets, (d) triplets, (f) quadruplets elicited with different IPIs as a function of muscle length category. Bars represent \pm S.E.M.

function of muscle length and IPI. The surfaces appear quite smooth and the changes in the pattern of summation continuous. When interpreting this figure, it should be kept in mind that each triplet was obtained by adding a pulse to the optimal doublet at that length, each quadruplet was obtained by adding a pulse to the optimal triplet at that length. The advantage of an extra pulse in the nFTIpP of an $n$-pulse sequence, must be evaluated by comparing it to the nFTIpP of the optimal $n-1$-pulse sequence on which it was built. The nFTIpP values of the op- timal doublets, triplets, and quadruplets at all length categories are shown in Fig. 6(a); it is clear that enhancement of FTIpP of the optimized pattern is much higher at low length. This results in a modified relationship between muscle length and normalized FTI of the contraction; Fig. 6(b) shows FTI for twitches, optimal doublets, optimal triplets, optimal quadruplets at all length categories normalized to the corresponding contractions at length category $10\left(\mathrm{nFTI}_{10}\right)$. The FTI of the optimal quadruplet at muscle category 1 is approximately $40 \%$ of the FTI of 


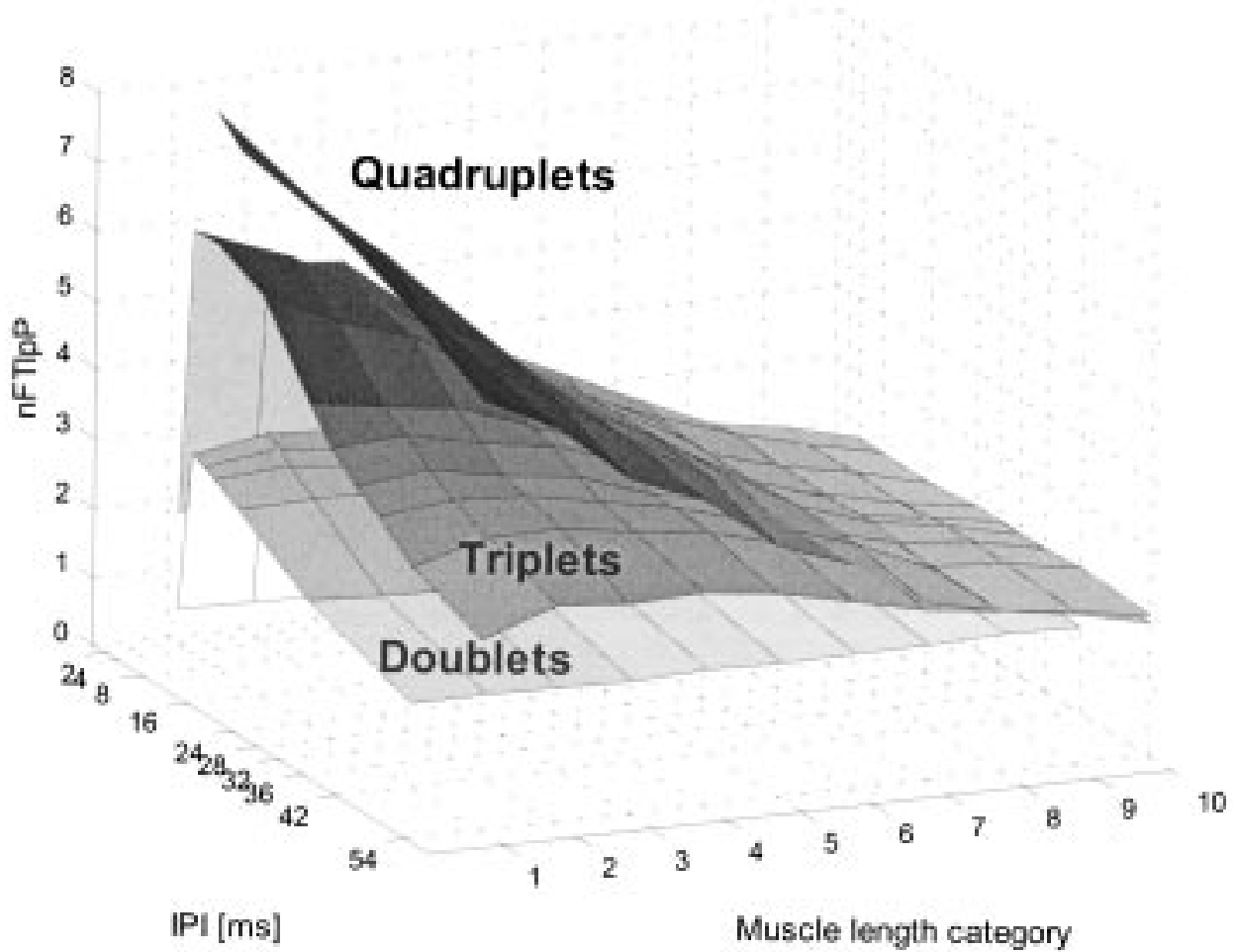

Fig. 5. Mean $\mathrm{nFTIpP}(n=6)$ for doublets (lower surface), triplets (intermediate surface), and quadruplets (upper surface) as a function of both muscle length and IPI. It should be remembered that, at each length category, triplets and quadruplets resulted from adding a pulse at varying IPIs to previously determined optimal doublets and triplets, respectively.

the optimal quadruplet at muscle length category 10 , while the FTI of a single twitch is only $15 \%$ of the FTI of the twitch at category 10 . When quadruplets are considered, the FTI of contractions elicited at category 5 and higher are above $80 \%$ of the value at category 10 , while for single twitches only category 9 is above the same relative value.

\section{DISCUSSION}

The purpose of this study was to determine the optimal four pulse stimulation sequence at different muscle lengths.

The results show that at high muscle length, the optimum stimulation pattern consists of an initial short IPI (doublet) followed by a longer IPI, in agreement with previous studies carried out at optimum muscle length [34], [24], and with the same muscle preparation [20]. nFTipP values at high muscle length are also consistent with those from previous research. However, $n F T I p P$ values increase with decreasing muscle length, being significantly higher at low length. Furthermore, it appears that, at low muscle length, a third stimulus after a second short IPI is also subject to more than linear summation, suggesting that the optimal pattern consists of a triplet of closely spaced pulses. Muscle length is thus shown to be an important factor that must be taken into account when specifying optimal stimulation patterns.

The biological systems underlying these effects can be thought of in two main categories. First, the length dependence of the efficacy of activation. Second, the length dependence of the ability to transmit force of the components of the muscle that link the force generators to the muscle tendon. We elimi- nated the effect of the extramuscular tendon by the method of preparation of the muscle.

The efficacy of activation could be defined as the time integral of the number of force-generating cross-bridges formed in response to a particular stimulus. A single action potential is thought to release sufficient $\mathrm{Ca}^{++}$to fully activate the contractile machinery ([15], [19]; however, see [11]). Since $\mathrm{Ca}^{++}$is rapidly pumped back to the sarcoplasmic reticulum, there may not be enough time for all activated cross-bridges to undergo attachment and force generation [28]. A second $\mathrm{Ca}^{++}$transient would then allow a longer time during which the myofilaments are exposed to high calcium concentrations. The magnitude of the effect of the second $\mathrm{Ca}^{++}$transient on force production might vary with muscle length because of the effect on cross-bridge interaction kinetics of length-dependent changes in interfilament lattice spacing [23]. It is possible that a closer position of the myosin heads to the actin binding sites due to reduced interfilament spacing at high muscle length, increases the rate at which the cross-bridge enters the force-producing state [7]. The time available for force production would then be less critical at high muscle length and the effect of a second $\mathrm{Ca}^{++}$transient smaller than at low muscle length. An increase in the attachment rate would result in a higher fraction of cycling cross-bridges in the force-generating state. The duration of isometric twitches elicited at increasing muscle length is longer and the ratio of the twitch peak force to the maximal tetanic force is larger confirming that force production in response to a single twitch is more effective at high length.

Another possible mechanism underlying the nonlinear summation of closely spaced twitches is the improved force trans- 


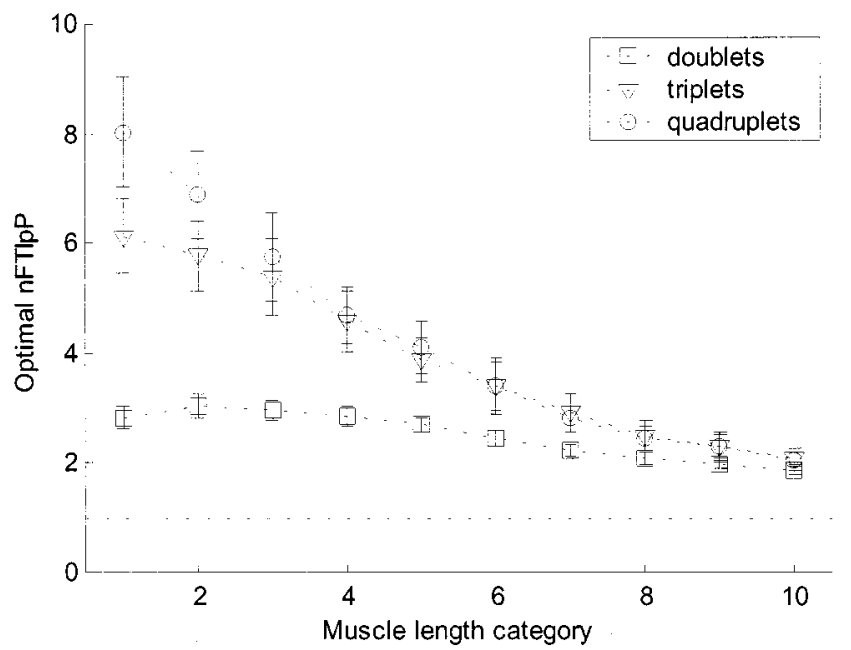

a)

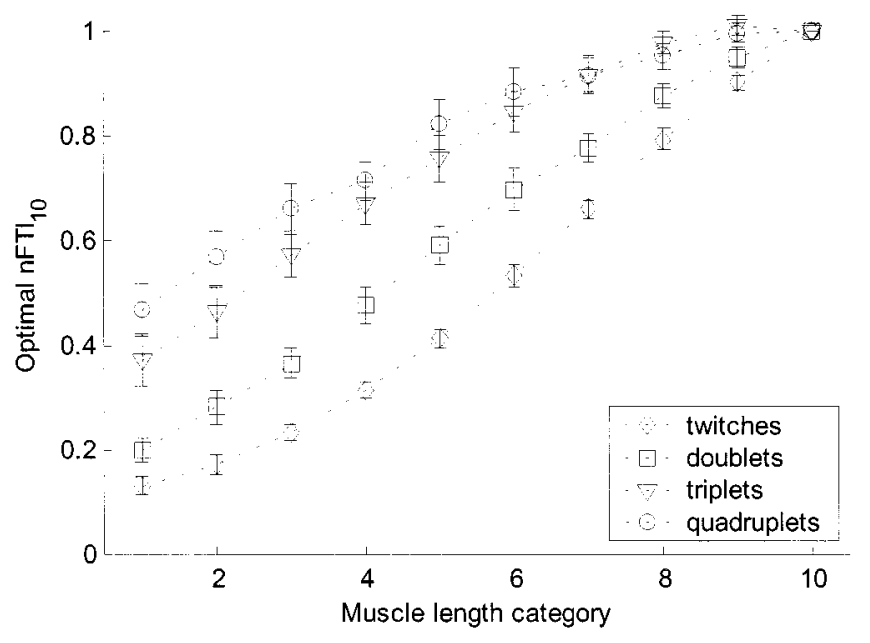

b)

Fig. 6. (a) Maximal nFTIpP \pm S.E.M. of doublets, triplets, and quadruplets at 10 muscle length categories. (b) FTI of twitches, optimal doublets, triplets, and quadruplets at 10 muscle length categories normalized to FTI of, respectively, twitches, optimal doublets, triplets, and quadruplets at length category 10 (optimal nFTI10)

mission due to the increased muscle-tendon complex stiffness produced by the first pulse. If the second twitch is delivered at a time when the muscle series elastic elements have been stretched by shortening due to the first pulse, force transmission will be improved. If this is a major mechanism underlying the doublet effect, then a smaller effect should occur at high muscle length as there may be little length change to be taken up by the initial pulse when the muscle is already passively stretched. For doublets, the optimum IPI was approximately $4 \mathrm{~ms}$ at all lengths, but the nFTIpP decreased significantly with increasing muscle length [Fig. 4(b)]. Although a single pulse could be enough to increase and maintain stiffness at high muscle length, two or more pulses may be required at low muscle length as force transmission may be hindered by the lack of time available to stretch the series elastic components. Binder-Macleod and
Lee [5] found that force enhancement with a stimulation pattern consisting of an initial doublet followed by a constant frequency portion with eight IPI of $70 \mathrm{~ms}$, occurred to a greater extend during isometric contractions than during concentric contractions, while little or no augmentation was observed during eccentric contractions. The authors concluded that part of the increased muscle stiffness produced by the initial doublet is lost as a result of muscle shortening during concentric contractions. Our observation that at low muscle lengths a third pulse is still beneficial in enhancing the muscle-tendon complex stiffness is consistent with this suggestion.

In this study we used the FTI of muscle response to stimulation as indicator of the functional output of the muscle. Many FES applications require a sustained fatigue-resistant force production over an active period. Since there is evidence that fatigue can be directly related to the total number of stimulation pulses received, practical FES should aim to produce the most force per stimulation pulse, hence FTIpP should be maximized.

Furthermore, reducing the number of pulses for a given force output might also be beneficial with respect to the energy expenditure associated with each stimulus at both the neuromuscular junction and for calcium cycling.

Studies to test the effect on force production of stimulation patterns taking advantage of an initial short IPI followed by a longer, constant IPI train have been performed on both healthy [6] and paralyzed muscles [12]. It appeared from both studies that such patterns did not result in a considerable improvement of the moment-time integral per pulse. Even though the patterns used in those studies contained an initial short IPI, they consisted of at least six pulses (six in [6], from 22 to 32 in [12]). The benefit of a fast rise in force on FTIpP declines when it is averaged over the duration of a long burst. Kwende et al. [20] showed that FTIpP of bursts with variable IPIs converge to that of a constant frequency burst with the same number of pulses when the number of pulses was more than five. Our results on quadruplets show that the fourth pulse did not improve the FTIpP, except at low muscle length.

To produce a sustained contraction, Karu et al. [18] used trains of stimulation consisting of sets of closely spaced stimulation bursts ( $n$-lets). They show that repeated doublets or triplets produced an enhancement of the force produced per stimulus pulse. It is then possible to obtain the same force profile in time with a much lower stimulus amplitude and, thus, compensate for the loss of force-generating capability of the muscle due to fatigue by increasing stimulation amplitude. In this way, the required force can be maintained for a longer period. Our study suggests that the repeated optimized pattern to be used in this way is muscle length dependent. Optimization of the FTI of the elicited contraction is a suitable criterion to obtain the optimal stimulation pattern. However, Karu et al. [18] indicated that practical applications of sustained contractions may require a relatively small force ripple, therefore, an appropriate repetition rate of the optimal sequence. Since three closely spaced pulses are required at low length, a relatively high repetition rate may be needed. This consideration leads to the conclusion that optimal patterns and the criterion used to determine them should be appropriately matched to the application, and studies should be performed using conditions similar to those of the actual applications. 


\section{ACKNOWLEDGMENT}

The authors wish to thank Dr. H. Sutherland for her skills in experimental preparations, and E. Droog and J. Blackhurst for their precious technical support.

\section{REFERENCES}

[1] W. S. al-Amood, A. J. Buller, and R. Pope, "Long-term stimulation of cat fast-twitch skeletal muscle," Nature, vol. 244, no. 5413, pp. 225-227, July 27, 1973.

[2] B. Bigland-Ritchie, D. A. Jones, and J. J. Woods, "Excitation frequency and muscle fatigue: Electrical responses during human voluntary and stimulated contractions," Exper. Neurol., vol. 64, no. 2, pp. 414-427, 1979.

[3] B. Bigland-Ritchie, I. Zijdewind, and C. K. Thomas, "Muscle fatigue induced by stimulation with and without doublets," Muscle Nerve, vol. 23, no. 9, pp. 1348-1355, 2000C.

[4] S. A. Binder-Macleod and C. B. Barker, III, "Use of a catchlike property of human skeletal muscle to reduce fatigue," Muscle Nerve, vol. 14, no. 9, pp. 850-857, 1991.

[5] S. A. Binder-Macleod and S. C. Lee, "Catchlike property of human muscle during isovelocity movements," J. Appl. Physiol., vol. 80, no. 6, pp. 2051-2059, 1996.

[6] S. A. Binder-Macleod, S. C. K. Lee, A. D. Fritz, and L. J. Kucharski, "New look at force-frequency relationship of human skeletal muscle: Effects of fatigue," J. Neurophysiol., vol. 79, no. 4, pp. 1858-1868, 1998.

[7] B. Brenner, "Effect of $\mathrm{Ca}_{2}{ }^{+}$on cross-bridge turnover kinetics in skinned single rabbit psoas fibers: Implications for regulation of muscle contraction," Proc. Nat. Acad. Sci. USA, vol. 85, no. 9, pp. 3265-3269, 1988.

[8] R. Burnham, T. Martin, R. Stein, G. Bell, I. MacLean, and R. Steadward, "Skeletal muscle fiber type transformation following spinal cord injury," Spinal Cord, vol. 35, no. 2, pp. 86-91, 1997.

[9] R. E. Burke, P. Rudomin, and F. E. Zajac, III, "The effect of activation history on tension production by individual muscle units," Brain Res., vol. 109 , no. 3, pp. 515-529, 1976.

[10] S. G. Carroll, R. J. Triolo, H. J. Chizeck, R. Kobetic, and E. B. Marsolais, "Tetanic responses of electrically stimulated paralyzed muscle at varying interpulse intervals," IEEE Trans. Biomed. Eng., vol. 36, pp. 644-653, July 1989.

[11] J. Duchateau and K. Hainaut, "Nonlinear summation of contractions in striated muscle. I. Twitch potentiation in human muscle," J. Muscle Res. Cell Motil., vol. 7, no. 1, pp. 11-17, 1986.

[12] H. M. Franken, P. H. Veltink, A. van Harn, and H. B. K. Boom, "The influence of a stimulation pattern on force and fatigue profiles in FESinduced quadriceps contractions in paraplegics," Basic Appl. Myol., vol. 4, pp. 173-186, 1994.

[13] tion," IEEE Eng. Med. Biol. Mag., vol. 13, pp. 564-570, Aug./Sept. 1994.

[14] S. J. Garland, S. H. Garner, and A. J. McComas, "Relationship between numbers and frequencies of stimuli in human muscle fatigue," J. Appl. Physiol., vol. 65, no. 1, pp. 89-93, 1988.

[15] J. M. Gillis, "Relaxation of vertebrate skeletal muscle. A synthesis of the biochemical and physiological approaches," Biochim. Biophys. Acta, vol. 811, no. 2, pp. 97-145, 1985.

[16] P. H. Gorman and J. T. Mortimer, "The effect of stimulus parameters on the recruitment characteristics of direct nerve stimulation," IEEE Trans. Biomed. Eng., vol. 30, pp. 407-414, July 1983.

[17] J. C. Jarvis, "Power production and working capacity of rabbit tibialis anterior muscles after chronic electrical stimulation at $10 \mathrm{~Hz}, " J$. Physiol., vol. 470, pp. 157-169, 1993.

[18] Z. Z. Karu, W. K. Durfee, and A. M. Barzilai, "Reducing muscle fatigue in FES applications by stimulating with N-let pulse trains," IEEE Trans. Biomed. Eng., vol. 42, pp. 809-817, Aug. 1995.

[19] M. Kress, H. E. Huxley, A. R. Faruqi, and J. Hendrix, "Structural changes during activation of frog muscle studied by time-resolved X-ray diffraction," J. Mol. Biol., vol. 188, no. 3, pp. 325-342, 1986.

[20] M. M. Kwende, J. C. Jarvis, and S. Salmons, "The input-output relations of skeletal muscle," Proc. Roy. Soc. Lond. B Biol. Sci., vol. 261, no. 1361, pp. 193-201, 1995.

[21] C. D. Marsden, J. C. Meadows, and P. A. Merton, "Muscular wisdom that minimizes fatigue during prolonged effort in man: Peak rates of motoneuron discharge and slowing of discharge during fatigue," $A d v$. Neurol., vol. 39, pp. 169-211, 1983.
[22] D. J. Maxwell, M. H. Granat, and R. H. Baxendale, "Novel stimulation strategies for the recruitment of paralyzed muscle," in Neuroprosthetics, A. Pedotti, M. Ferrarin, J. Quintern, and R. Riener, Eds: Springer-Verlag, 1996, pp. 115-122

[23] K. S. McDonald, M. R. Wolff, and R. L. Moss, "Sarcomere length dependence of the rate of tension redevelopment and submaximal tension in rat and rabbit skinned skeletal muscle fibers," J. Physiol., pt. 3, vol. 501, pp. 607-621, 1997.

[24] F. Parmiggiani and R. B. Stein, "Nonlinear summation of contractions in cat muscles. II. Later facilitation and stiffness changes," J. Gen. Physiol., vol. 78, no. 3, pp. 295-311, 1981.

[25] P. M. Rack and D. R. Westbury, "The effects of length and stimulus rate on tension in the isometric cat soleus muscle," J. Physiol. (Lond.), vol. 204, pp. 443-460, 1969.

[26] F. Rattay, "Modeling the excitation of fibers under surface electrodes," IEEE Trans Biomed. Eng., vol. 35, no. 3, pp. 199-202, 1988.

[27] N. J. Rijkhoff, H. Wijkstra, P. E. van Kerrebroeck, and F. M. Debruyne, "Urinary bladder control by electrical stimulation: Review of electrical stimulation techniques in spinal cord injury," Neurourol. Urodyn., vol. 16, no. 1, pp. 39-53, 1997.

[28] J. C. Rüegg, Calcium in Muscle Contraction. Heidelberg, Germany: Springer-Verlag, 1992, pp. 74-80.

[29] S. Salmons and J. C. Jarvis, "Cardiac assistance from skeletal muscle: A critical appraisal of the various approaches," Brit. Heart J., vol. 68, no. 3, pp. 333-338, 1992.

[30] G. Sjogaard, G. Savard, and C. Juel, "Muscle blood flow during isometric activity and its relation to muscle fatigue," Eur. J. Appl. Physiol. Occup. Physiol., vol. 57, no. 3, pp. 327-335, 1988.

[31] H. Sutherland, J. C. Jarvis, M. M. Kwende, S. J. Gilroy, and S. Salmons, "The dose-related response of rabbit fast muscle to long-term low-frequency stimulation," Muscle Nerve, vol. 21, no. 12, pp. 1632-1646, 1998.

[32] P. N. Taylor, J. H. Burridge, A. L. Dunkerley, D. E. Wood, J. A. Norton, C. Singleton, and I. D. Swain, "Clinical use of the Odstock dropped foot stimulator: Its effect on the speed and effort of walking," Arch. Phys. Med. Rehab., vol. 80, no. 12, pp. 1577-1583, 1999.

[33] P. H. Veltink and N. Donaldson, "A perspective on the control of FESsupported standing," IEEE Trans. Rehab. Eng., vol. 6, pp. 109-112, June 1998.

[34] F. E. Zajac and J. L. Young, "Properties of stimulus trains producing maximum tension-time area per pulse from single motor units in medial gastrocnemius muscle of the cat," Neurophysiol., vol. 43, no. 5, pp. 1206-1220, 1980.

Petra Mela received the M.Sc. degree in mechanical engineering from the Politecnico di Torino, Torino, Italy, in 1996 and the Ph.D. degree from the University of Twente, Enschede, The Netherlands, in 2001. The research performed toward the Ph.D. degree involved muscle dynamics during neuromuscular stimulation and was carried out within the Institute for Biomedical Technology.

She is currently with the MESA ${ }^{+}$Research Institute at the University of Twente where she is involved in research on flow control in micro-nanofluidic systems.

Peter H. Veltink ( $\mathrm{S}^{\prime} 85-\mathrm{M}^{\prime}$ '88) studied electrical engineering at the University of Twente, Enschede, The Netherlands, and performed Ph.D. research in the area of nerve stimulation for functional electrical stimulation.

$\mathrm{He}$ is a Professor of Technology for the Restoration of Human Function at the Institute for Biomedical Technology, University of Twente, and performs research in the area of artificial motor control with applications to rehabilitation medicine. He was on sabbaticals at Case Western Reserve University, Cleveland, OH, in 1989 and at the Center for Sensory-Motor-Interaction at Aalborg University, Aalborg, Denmark, in 1997. He (co)authored 47 publications in international reviewed scientific journals and over 140 conference papers. He was Editor of three volumes of conference proceedings.

Prof. Veltink is involved and coordinates several European projects and was Treasurer of the International Functional Electrical Stimulation Society (IFESS). He received the Royal Shell Stimulating Prize for his contribution to the rehabilitation-engineering field in 1997. 